\title{
Ressourcenverfügbarkeit und Bedarfsplanung auf Basis geänderter Rahmenbedingungen
}

\author{
Roman Neunteufel · Benedikt-Johannes Schmidt · Reinhard Perfler
}

Online publiziert: 24. März 2017

C Der/die Autor(en) 2017. Dieser Artikel ist eine Open-Access-Publikation.

\begin{abstract}
Zusammenfassung Die Herausforderung für die Zukunft ist es, unter gleichzeitiger Berücksichtigung der sozioökonomischen und demografischen Entwicklung und des Klimawandels, die nötigen Maßnahmen zur Erhaltung der Versorgungssicherheit vorausschauend $\mathrm{zu}$ planen und umzusetzen. Insbesondere betrifft das die in Zukunft benötigten Wasserressourcen.
\end{abstract}

In Bezug auf die Sicherstellung der Wasserversorgung wirkt der Klimawandel auf verschiedenen Ebenen beeinflussend:

- Auf der Konsumentenseite steigt der Verbrauch in Trocken- und Hitzeperioden deutlich an, sodass zur Abdeckung des Spitzenwasserverbrauchs zusätzliche Ressourcen oder Anlagen nötig werden können.

- Auf der Ressourcenseite kann sich durch eine zunehmende Verschiebung der Niederschläge das Dargebot verändert darstellen (z. B. kann es durch Niederschlagsdefizite im Sommer und Herbst zu Wasserknappheit in Wintertourismusregionen im Westen Österreichs kommen).

- Durch konkurrierende Wasserentnahmen (z. B. Bewässerungsbedarf der Landwirtschaft in der trockener werdenden Vegetationsperiode) kann ein zusätzlicher Nutzungsdruck in quantitativer und qualitativer Hinsicht entstehen.

Aufgrund der Langlebigkeit und langsamen Anpassung der Wasserinfrastruktur gilt es, ein breites Wissen über die möglichen Szenarien zur Überprüfung der Planungsansätze verfügbar zu haben. Eine regional integrierte Wasserversorgungsplanung soll daher insbe-

DI Dr. R. Neunteufel $(\varangle)$.

DI B.-J. Schmidt .

Priv.-Doz. DI Dr. R. Perfler

Institut für Siedlungswasserbau,

Industriewasserwirtschaft und

Gewässerschutz, Universität

für Bodenkultur Wien,

Muthgasse 18, 1190 Wien, Österreich

roman.neunteufel@boku.ac.at sondere eine Ressourcennutzungsplanung mit Bestandsaufnahmen verfügbarer Ressourcen und Prognosen des zukünftigen Wasserbedarfs umfassen.

Resource availability and needs planning on the basis of changed framework conditions

Abstract The great challenge for the future consists in planning and implementing necessary measures to ensure reliability of supply, while at the same time taking into account socioeconomic, demographic and climate change. This is particularly relevant for the water resources that will be needed in the future.

With regard to ensuring the future water supply, the impacts of climate change can be felt at various levels:

- Regarding consumers, water consumption will rise significantly in dry and hot periods. As a result, additional resources and/or facilities may be needed in order to cover peak demand periods.

- Regarding resources, shifts in precipitation could lead to changes in resource-yields (e.g. reduced rainfall in the summer and autumn could lead to water shortages in Western Austria's winter tourism regions).

- Competing water needs (e.g. the need for agricultural irrigation in increasingly dry periods) could result in additional (quantitative and qualitative) pressure on the waterresources.

Given the longevity and slow adaptation of the water infrastructure, the goal is now to establish a broad range of information on potential scenarios for reviewing various planning approaches. As such, regionally integrated water-supply planning should above all encompass resource-use planning, including evaluation of available resources and projections of future water needs.

\section{Hintergrund und Zielsetzung}

Da Anpassungsmaßnahmen im Infrastrukturbereich erfahrungsgemäß zum Teil eine sehr lange Vorlaufzeit haben, die je nach Maßnahmenkategorie auch bis zu 25 oder 30 Jahren betragen kann (z. B. Wasserschutzgebiete), wurde im Auftrag der ÖVGW (Österreichische Vereinigung für das Gas- und Wasserfach) eine Studie beauftragt, in der die zukünftigen Rahmenbedingungen der Wasserversorgung beleuchtet wurden.

Insbesondere wurden Zusammenhänge der Ressourcenverfügbarkeit und des Wasserbedarfs mit Hinblick auf den Klimawandel, aber auch in Bezug auf sozio-ökonomische Veränderungsprognosen untersucht. Als Referenzszenarien für mögliche zukünftige Wetterlagen wurden die Rekordsommer der Jahre 2003 und 2015 herangezogen, um die Versorgungssituation unter langandauernden Trocken- und Hitzeperioden zu beurteilen und Handlungsoptionen darzustellen.

Ziel der Studie war es unter anderem festzustellen, wie gut die Versorgungssicherheit in den bisherigen Extremsommern war, ob in Zukunft häufiger mit derartigen Situationen gerechnet werden muss und welchen Einfluss die geänderten Rahmenbedingungen auf die Wasserversorgungssituation haben können.

Die vorliegende Arbeit stellt eine $\mathrm{Zu}$ sammenfassung der Studienergebnisse mit Fokus auf die Ressourcenverfügbarkeit und Bedarfsplanung dar (Neunteufel et al. 2016).

\section{Methodik}

Die Fragestellungen der Studie wurden grob in folgende Kategorien eingeteilt:

1. Hintergrundinformationen $\mathrm{zu}$ Klima, Demografie, Sozioökonomie und Verbraucherverhalten,

2. Überlegungen zu quantitativen Aspekten der Wasserversorgung, 
3. Kennzahlen zu quantitativen Aspekten der Wasserversorgung,

4. Überlegungen zu Aspekten der Wasserqualität,

5. Kennzahlen zur Wasserqualität,

6. Zusammenschau und Resilienzbetrachtung - Ist-Zustand/mögliche zukünftige Szenarien,

7. Handlungsoptionen „Armaturenbrett - was wirkt wie?" und

8. Defizitanalyse - unsichere fehlende Informationen.

Zur Erarbeitung der Fragestellungen wurden folgende Informationsquellen herangezogen: Zusammenfassung

a) und Auswertung existierender Studien, Veröffentlichungen und verfügbarer Datengrundlagen.

b) Erhebung der Versorgungssituation durch eine Umfrage, die von der ÖVGW sowie dem BMLFUW bzw. über die Landesregierungen an die Wasserversorger ausgesendet wurde. Die Umfrage umfasste sowohl qualitative Aussagen als auch quantifizierbare Kennzahlen.

c) Experteninterviews bei den Landesregierungen.

Hinsichtlich der verwendeten sehr umfangreichen Datenquellen sei an dieser Stelle noch einmal auf die Studie verwiesen (Neunteufel et al. 2016).

\section{Ergebnisse}

\subsection{Veränderungen der Rahmenbedingungen}

\subsubsection{Einflussfaktoren auf den Wasserverbrauch}

Bei den Auslösern für die Variabilität des Wasserverbrauchs kann in langfristig und kurzfristig wirkende Einflussfaktoren unterschieden werden.

Langfristig existierende Verbrauchsunterschiede hängen im Wesentlichen von den Verbrauchergruppen innerhalb der jeweiligen Siedlungsstruktur (ländliche/städtische Siedlungsgebiete) und von sozioökonomischen Parametern ab (aufstrebende Zuwanderungsgemeinden im „Speckgürtel“ von Ballungsräumen/Abwanderungsgebiete).

Die kurzfristige Variabilität des Verbrauchs wird hingegen maßgeblich vom Wetter sowie dem Wochentag und der Tageszeit beeinflusst. Wie bereits im Forschungsprojekt „Wasserverbrauch und Wasserbedarf" (WAVE-
Studie, Neunteufel et al. 2013) beschrieben wurde, führen längere trockene Perioden bei gleichzeitig hohen Temperaturen $\mathrm{zu}$ einem starken Ansteigen der Tageswasserverbräuche. Im Mittel wurde eine knappe Verdopplung des durchschnittlichen Tagesverbrauchs gegenüber dem Verbrauch an kühlen Tagen außerhalb von Trockenperioden festgestellt. Individuelle Spitzenfaktoren einzelner WVU liegen noch weitaus höher.

Die Variabilität des Verbrauchs aufgrund der kurzfristigen Einflussfaktoren übersteigt jene von langfristig wirkenden Faktoren jedenfalls deutlich und ist maßgeblich für die Spitzenfaktoren (Tages- und Stundenfaktoren) verantwortlich.

Einige Einflussfaktoren treten in unterschiedlich starker Ausprägung täglich oder wöchentlich auf (Tagesganglinie, Wochenganglinie), andere sind saisonal bedingt und wetterabhängig. Die jeweiligen Wassernutzungsarten sind dabei unterschiedlich: Während die Morgenspitze der Tagesganglinie vorwiegend durch WC und Körperhygiene ausgelöst wird, sind die wetterabhängigen Verbrauchsspitzen auf den Außenverbrauch für Bewässerung und private Swimmingpools zurückzuführen. Von einer grundsätzlichen zeitlichen Koinzidenz der Spitzenauslöser innerhalb eines Haushalts ist dabei zwar nicht auszugehen, die Zeitpunkte von individuellen Verbrauchsspitzen verschiedener Haushalte in einem Versorgungsgebiet weisen aber zumindest zum Teil eine gewisse Gleichzeitigkeit auf.

Während Stundenspitzen maßgeblich für die Leitungsnetzdimensionierung sind und über eine entsprechende Behälterkapazität ausgeglichen werden, sind die maximalen Tageswasserverbräuche hinsichtlich der Ressourcenkapazität relevant.

\subsubsection{Szenarien für die klimatische Entwicklung}

Die klimasteuernden Parameter sind die Sonnenaktivität, deren Minima sich oft als kältere Perioden in Klimaarchiven auffinden lassen, Vulkanausbrüche, die durch emittierten Staub und Gase zu Abkühlungen (Abschattungseffekte) führen, und die Treibhausgaskonzentration.

Die atmosphärische Strahlungsbilanz wird einerseits durch Abschattungseffekte (Global Dimming, z. B. durch Rußpartikel und Staub) beeinflusst, die die Sonneneinstrahlung vermindern und $\mathrm{zu}$ einem Temperaturrückgang führen. Andererseits vermindern die Treibhausgaskonzentrationen in der Atmosphäre die Wärmeabstrahlung zurück in den Weltraum, wodurch eine Temperaturerhöhung eintritt.

Gase in der Atmosphäre, die wesentlich zum Treibhauseffekt beitragen, sind Wasserdampf, Kohlendioxid und Methan.

Wasserdampf besitzt aufgrund der großen Mengen in der Atmosphäre die stärkste direkte Treibhauswirkung. Die Wasserdampfkonzentration ist im Wesentlichen von der Verdunstung aus den Ozeanen abhängig und wird somit direkt von der globalen Erwärmung beeinflusst.

Kohlendioxid ist in der Atmosphäre derzeit mit einem Anteil von rund $0,04 \%$ vertreten. Diese Konzentration trägt zu maximal einem Viertel der Treibhauswirkung bei. Der aktuell beobachtbare Anstieg der Kohlendioxidkonzentration in der Atmosphäre ist vorwiegend auf die Verbrennung fossiler Brennstoffe zurückzuführen.

Der Methangehalt der Atmosphäre ist zwar wesentlich geringer als der $\mathrm{CO}_{2}$ Gehalt, aufgrund seiner vielfach stärkeren Treibhauswirksamkeit trägt Methan aber auch in vergleichbarem Ausmaß zur Treibhauswirkung bei. Methan wird in der Landwirtschaft, insbesondere der Tierproduktion sowie aus stehenden Wasserflächen wie z. B. Reisfeldern freigesetzt. Ein weiterer großer Anteil des Methans in der Atmosphäre entstammt aus Verlusten bei der Förderung, dem Transport und der Verarbeitung von Erdgas. Der globale mittlere Methangehalt der Atmosphäre hat sich durch anthropogene Einflüsse seit den vorindustriellen Zeiten mehr als verdoppelt. Große Mengen von Methan sind (noch) in den Meeren als Methanhydrat gespeichert (APCC 2014; Solomon et al. 2007).

Hintergrundrecherchen $\mathrm{zu}$ Klimamodellen haben gezeigt, dass der Alpenraum vom Klimawandel dabei noch stärker betroffen sein wird als der globale Durchschnitt. Die fortschreitende Klimaveränderung wird höhere Durchschnittstemperaturen und voraussichtlich häufigere Hitzeperioden mit sich bringen und erscheint aus heutiger Sicht zumindest kurz- und mittelfristig unvermeidlich (APCC 2014).

Der Klimawandel ist eine belegte Tatsache und wird die zukünftige $\mathrm{Si}$ - 
cherstellung der Wasserversorgung auf verschiedenen Ebenen beeinflussen. Die Rekordsommer der vergangenen Jahre, speziell 2003 und 2015, können gleichsam als Referenz für eine mögliche zukünftige Versorgungssituation herangezogen werden.

Hinsichtlich der Einflussfaktoren auf den Wasserverbrauch ist jedenfalls das Auftreten von Trockenperioden und Hitzewellen in den Sommern von Interesse. Diesbezüglich lassen die derzeitigen Klimamodelle eine starke Zunahme der Hitzetage erwarten.

Aufgrund der auftretenden Veränderungen wird darüber hinaus hinterfragt, ob sich die ursprünglich angenommenen Wahrscheinlichkeiten der Wiederkehrhäufigkeiten von Extremwetterlagen aufrechterhalten lassen. Zum Beispiel wurde die Hitzewelle 2003 noch als ein 200-jährlicher Extremfall eingestuft. Gleichzeitig bestehen aber Annahmen aus Modellen, dass derartige Hitzewellen in den nächsten 40 Jahren um 5 - bis 10-mal häufiger auftreten werden.

Ein Hitzetage-Szenario für Österreich wurde im Forschungsprojektes COIN (Formayer et al. 2015, zit. in Steininger et al. 2015) erstellt. Daraus ist ersichtlich, dass die Anzahl der Hitzetage (vereinfacht: eine Serie von mind. drei Tagen mit Höchsttemperaturen über $30^{\circ} \mathrm{C}$ ) zum Beispiel in Teilen Niederösterreichs von „heute“ 10 bis 15 auf 30 bis 35 in der Periode um 2050 ansteigen kann. Der mögliche Anstieg, zum Beispiel in Kärnten, beträgt von „heute“ 15 bis 20 auf 35 bis 40 in der Periode um 2050 und auf über 50 Hitzetage in der Periode um 2085. Die Anzahl der Hitzetage entspricht dabei der für die Periode typischen Anzahl. „Heute“ steht für die Referenzperiode 1981 bis 2010, „2030“ = Periode 2016 bis 2045, „2050“ = Periode 2036 bis 2065 und „2085“ = Periode 2071 bis 2100 .

Inwiefern sich die Wetterlagen unter dem Klimawandel entwickeln werden, kann aus heutiger Sicht noch nicht zufriedenstellend vorhergesagt werden. Nach derzeitigem Wissensstand ist es wahrscheinlich, dass sich der subtropische Hochdruckgürtel im Sommer weiter in den Norden ausbreitet. Damit könnten Wetterlagen mit sehr heißem und trockenem Wetter, die jetzt für den Mittelmeerraum typisch sind, auch für Mitteleuropa häufiger werden. Wie sich das Wetter in der Zukunft von Jahr zu Jahr entwickeln wird, hängt von der genauen Lage der Hochdruckgebiete ab. Zum Beispiel ist ein heißer und tro- ckener Sommer wie 2015 ebenso ein mögliches Szenario wie der Sommer 2012, der in Österreich zahlreiche Unwetter brachte (Haslinger et al. 2015).

Entsprechend der erwarteten Klimaänderung kann zusammengefasst werden, dass

- sich mit den Veränderungen der mittleren Bedingungen (z. B. höhere Jahresmitteltemperatur) auch Häufigkeit und Intensität von Spitzentemperaturen verändern werden,

- eine Zunahme der Extremereignisse, insbesondere der Hitzewellen und Trockenperioden, wahrscheinlich ist und

- ein höheres Temperaturniveau auch höhere Feuchtegehalte der Luft bedingt und Starkniederschläge, wenngleich nicht unbedingt häufiger, aber intensiver ausfallen könnten.

In Hinblick auf die Wasserversorgung ist festzuhalten, dass jede Erhöhung der Variabilität des Wetters - hin zu längeren Trockenperioden, mehr Hitzewellen und intensiveren Niederschlagsereignissen sowie die Abnahme des Schneeniederschlages durch den Temperaturanstieg und der Rückgang der Sommerniederschläge - fast ausschließlich ungünstige Auswirkungen auf die Versorgungssituation haben wird.

\subsubsection{Demografische und sozioökonomische Entwicklung}

Die Siedlungs- und Stadtentwicklung ist entsprechend den lokalen Gegebenheiten sehr unterschiedlich. Verfügbarkeit und Leistbarkeit von Siedlungsfläche und Wohnraum sowie der Arbeitsmarkt sind die maßgeblichen Faktoren.

Die österreichweit wahrscheinlichsten demografischen und sozioökonomischen Entwicklungen wurden im Forschungsprojekt COIN (Steininger et al. 2015) in einem „Middle of the road“Szenario zusammengefasst.

Demnach wird die Bevölkerung ausgehend von 8,4 Millionen Einwohnern in 2011 auf 9,0 Millionen im Jahr 2030 und 9,3 Millionen in 2050 anwachsen. Der Anteil älterer Menschen über 65 Jahre wird von $18 \%$ in 2011 auf $25 \%$ in 2030 und $28 \%$ in 2050 steigen. Dadurch steigt das Durchschnittsalter der Bevölkerung um 4 Jahre.

Wanderungsbewegungen in die Städte werden weiterhin erhalten bleiben. Je größer und zentraler die Städte sind, desto stärker wird der Zuzug in diese Gebiete sein. Der Zuzug wird aus wirt- schaftlich schwächeren Räumen auf nationaler Ebene und aus dem EU-Ausland erfolgen. Durch die abnehmende Haushaltsgröße wird zunehmend mehr Wohnfläche benötigt. Der Landverbrauch und die damit verbundenen technischen Infrastrukturen werden dementsprechend ebenso wachsen, wenngleich ein Teil des Zuzugs durch eine Erhöhung der Bevölkerungsdichte aufgenommen wird und sich nicht 1:1 im Gebäudebestand niederschlägt.

Durch die sinkende klimatische Lebensqualität in dicht verbauten Gebieten (urbane Hitzeinseln) werden jene Menschen, die dazu wirtschaftlich in der Lage sind, verstärkt in das Umland der großen Städte ziehen. Die Siedlungsflächen werden in den kommenden Jahrzehnten daher speziell rund um die Städte (Speckgürtel) weiterhin deutlich zunehmen.

Die Siedlungsflächen in wirtschaftlich schwachen Regionen werden zwar weitgehend unverändert bleiben, die Bevölkerungsdichte der dauerhaft ansässigen Personen wird aber abnehmen. Die gelegentliche Nutzung als Zweitwohnsitz oder Vermietung an Feriengäste wird stärker werden.

Die Ergebnisse der für die Studie durchgeführten Umfrage unter den Wasserversorgern zeigen, dass bis zum Jahr 2050 von den Wasserversorgern im Schnitt eine Zunahme von 20 \% erwartet wird. Einzelne maximale Schätzungen reichen bis zu einer Verdopplung der versorgten Einwohner. In absoluten Zahlen betrachtet sind die Bevölkerungszunahmen der urbaneren und dadurch zumeist auch größeren Versorgungsgebiete natürlich deutlich höher als die der ländlichen Gebiete.

Dass gerade auch in den ländlichen Versorgungsstrukturen $\mathrm{z}$. T. hohe $\mathrm{Zu}$ wachsraten erwartet werden, könnte darin begründet sein, dass es sich einerseits um Umlandgemeinden der Ballungszentren handelt, sowie, dass zunehmend mit einem Anschluss von bisherigen Einzelversorgungen an die öffentlichen Versorgungssysteme gerechnet wird.

Die künftige Bevölkerungsentwicklung Österreichs auf regionaler Ebene kann den jeweils aktuellen Veröffentlichungen der Statistik Austria bzw. der ÖROK (Österreichische Raumordnungskonferenz) entnommen werden.

Entsprechend der erwarteten sozioökonomischen Veränderungen kann zusammengefasst werden, dass 
- die individuellen Bevölkerungsprognosen jedenfalls eine wesentliche Grundlage sowohl für die Erweiterungs- und Erneuerungsplanung der Leitungsinfrastrukturen und Behältergrößen wie auch für die Beurteilung der zukünftig notwendigen Ressourcenkapazitäten sind, und

- speziell in einkommensstärkeren Regionen und Siedlungsstrukturen mit Eigengärten die Auswirkungen einer Zunahme von privaten Swimmingpools und die Gartenbewässerung von wesentlicher Bedeutung sein werden.

\subsection{Ressourcensituation}

\subsubsection{Entwicklung der} Ressourcenquantität

Eine wesentliche Auswirkung auf die Versorgungssicherheit könnte sich durch klimabedingte Veränderungen von Niederschlägen ergeben. Österreichweit und durchschnittlich betrachtet wird zwar nicht von einem generellen Wassermangel ausgegangen, sinkende Grundwasserneubildungsraten und Quellschüttungen könnten jedoch die lokale Ressourcenverfügbarkeit deutlich einschränken und so auch die Trinkwasserversorgung in Bedrängnis bringen (APCC 2014).

Das EU-Projekt „CC-WaterS“ (2012) nennt neben den direkten Auswirkungen des Klimawandels auf die Grundwasserneubildung auch Veränderungen in der Landnutzung als Treiber, die den Druck auf die Wasserressourcen und somit auf die öffentliche Trinkwasserversorgung verstärken.

Die im Rahmen des Projektes CCWaterS untersuchten hydrologischen Auswirkungen auf Quellschüttungen basierten auf Modellen unter Verwendung des gängigen A1B-Emissionsszenarios, wiesen aber große Unsicherheiten für den Niederschlag auf. Durch die höheren Temperaturen fällt im Winter jedenfalls weniger Niederschlag in Form von Schnee. Die Abflüsse im Winter nehmen zu, während die Sommerabflüsse abnehmen und die saisonale Entwicklung des Abflusses weist in Zukunft auf eine zeitlich frühere Abflussspitze hin (CC-WaterS 2012). Eine frühere Abflussspitze wäre auch mit einem früheren Rückgang der Quellschüttungen verbunden. Hinsichtlich der Verbrauchscharakteristik bzw. der jeweils typischen Zeitpunkte der Verbrauchsspitzen eines Versorgungsgebiets ist diese Verschiebung der Maxima und Minima des Ressourcendargebots von Bedeutung.

Bei der Betrachtung der Ressourcensituation muss jedenfalls eine Unterscheidung nach der Art der Grundwasserkörper (Karst-, Kluft- und Porengrundwasser) vorgenommen werden.

Die stärksten Reaktionen auf Witterungsänderungen zeigen im Allgemeinen Karstquellen. Daher war der Rekordsommer 2003 bei dieser Art der Quellen deutlich bemerkbar. Vor al- lem bei oberflächennahen Karstquellen in den nördlichen und südlichen Kalkalpen kam es zu einem merkbaren Rückgang der Ergiebigkeit.

Kluftgrundwässer (z. B. in geklüftetem Mergel und im Kristallin der Alpen) sind meist wechselnd bis gering ergiebig und oberflächennahe Quellfassungen reagieren ähnlich wie Karstquellen sehr stark auf Trockenheit. Derartige Quellen können für die Trinkwasserversorgung leicht ausfallen.

Die Porengrundwasserkörper reagierten sehr unterschiedlich auf die Trockenheit der Rekordsommer.

Bei den Grundwasserkörpern, die mit Flüssen eines nivo-glazialen $\mathrm{Ab}$ flussregimes (durch Schnee- und Gletscherschmelzwasser gespeist) in Kontakt stehen (z. B. Isel, Drau, Inn) waren die Auswirkungen des Trockenjahres 2003 durch die erhöhten Temperaturen nicht so dramatisch. Die Auswirkungen auf Grundwasserkörper, die mit Flüssen in Verbindung stehen, die kein vergletschertes Einzugsgebiet aufweisen, waren im Laufe des Sommers 2003 schon wesentlich stärker ausgeprägt. WVU, die von Auswirkungen des Rekordsommers 2003 auf ihre Ressourcensituation betroffen waren, waren vor allem Klein- und Kleinstversorger, die nicht über redundante Gewinnungsanlagen verfügten, sondern oft nur über einzelne Quellen bzw. oberflächennahe Brunnen. Zu Engpässen bei größeren WVU kam es vorwiegend in Fällen, wo

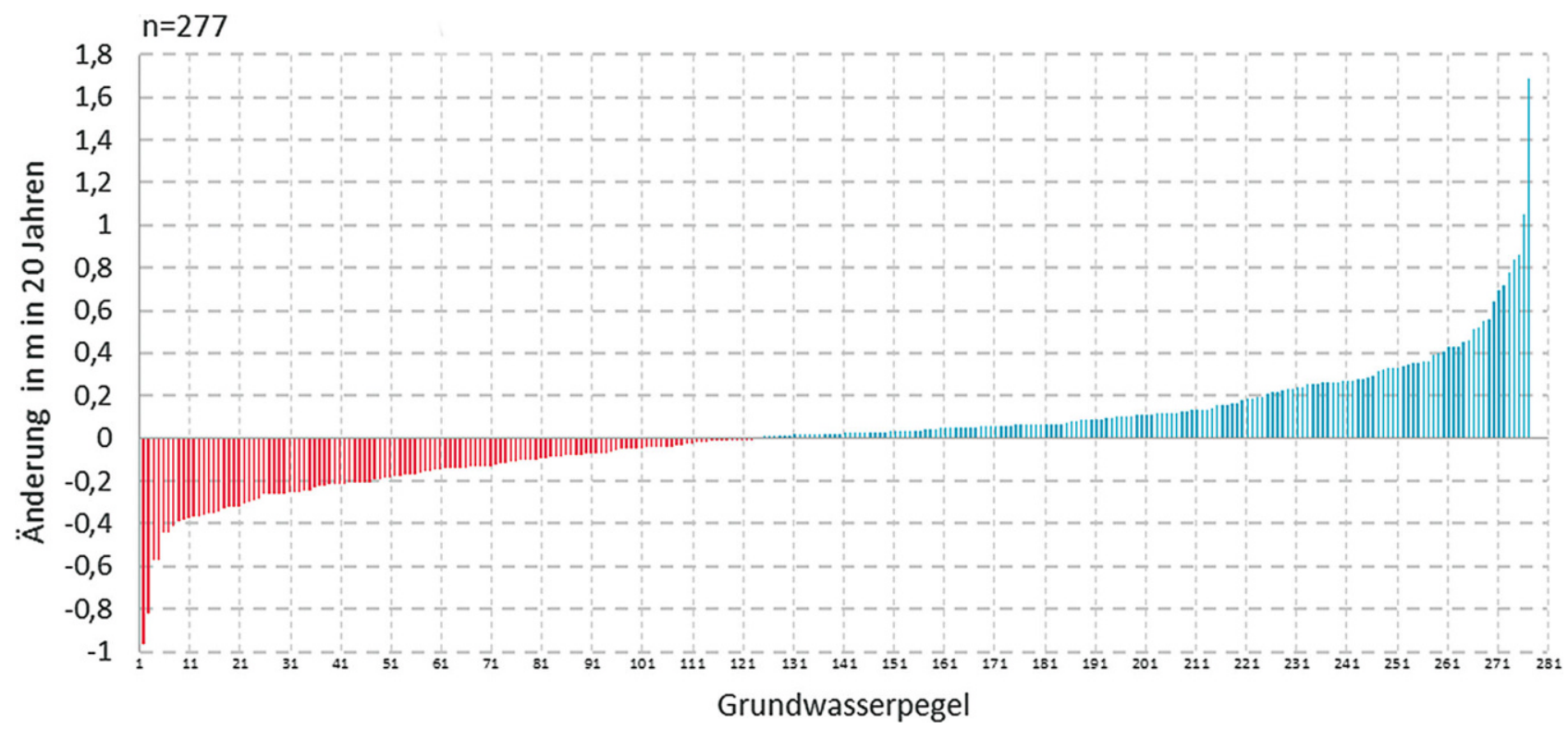

Abb. 1 Entwicklung der Grundwasserstände in m pro 20 Jahren (Daten: eHYD2015, Datenbasis jeweils mind. 20 Jahre, eigene Darstellung) 


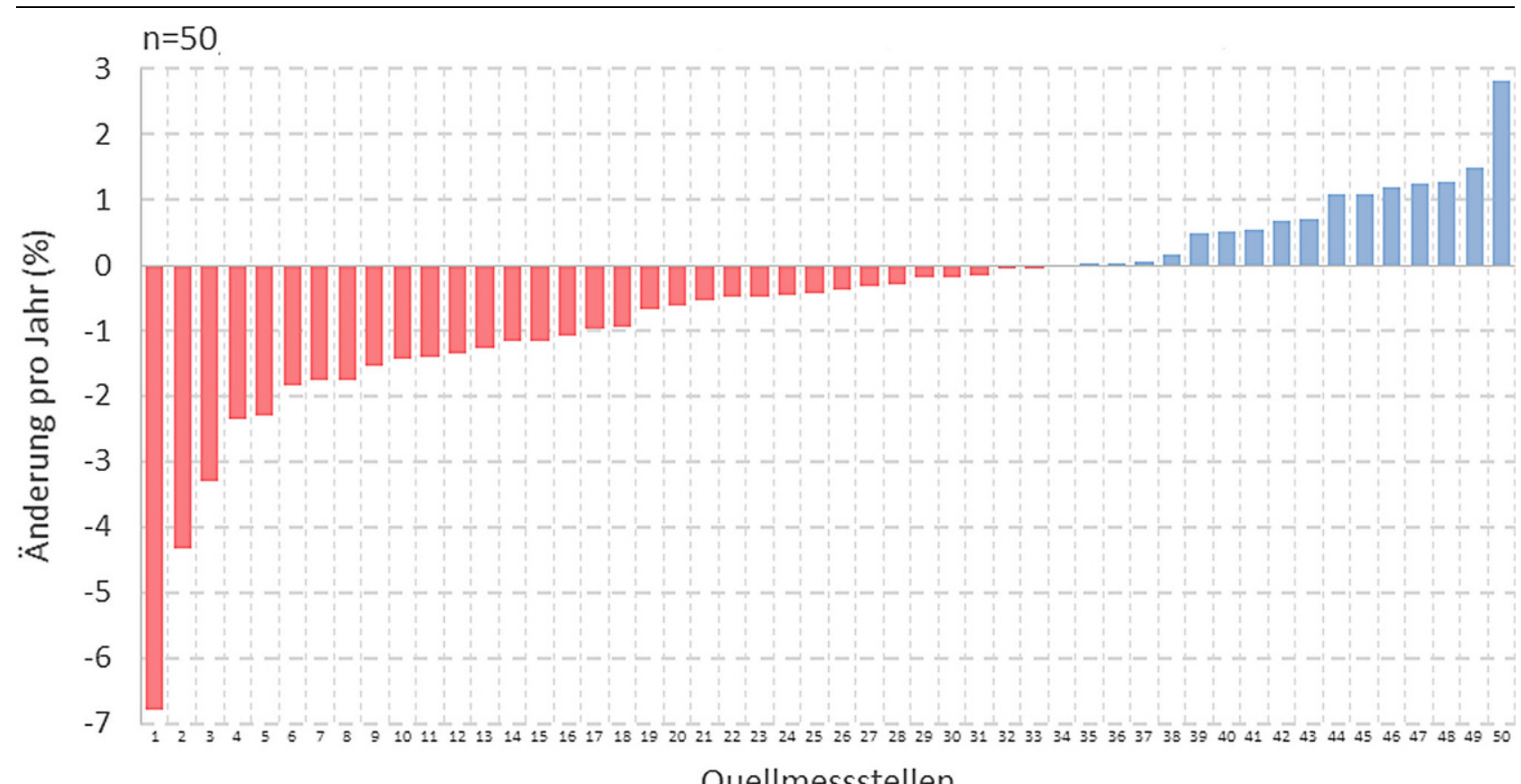

Abb. 2 Entwicklung der Quellschüttungen in \% pro Jahr(Daten: eHYD2015, Datenbasis jeweils mind. 10 Jahre, eigene Darstellung)

hauptsächlich Quellwasser als Ressource genutzt wurde (Perfler et al. 2006).

Hinsichtlich der glazial beeinflussten Abflussregime ist anzumerken, dass der fortschreitende Massenverlust der Gletscher zukünftig zu einer immer weiter abnehmenden Bedeutung der Ressourcendotation durch die Eisschmelze in den Sommermonaten führen wird.

Eine weitere Folge könnte sein, dass die Grundwasserkörper aufgrund der geringen Ergiebigkeit der Schneeschmelze bereits früher im Jahr ihre Maximalstände erreichen und Anfang Sommer bereits wieder zurückgehen. Bei mehreren aufeinander folgenden Rekordsommern könnten somit neue und noch niedrigere Grundwasserstände erreicht werden. Dies ist zwar nur ein Szenario, die Wahrscheinlichkeit von früheren Maximalständen und früheren Rückgängen der Grundwasserstände im Jahresverlauf erhöht sich aber mit steigenden (Winter-)Temperaturen, geringeren Schneeniederschlägen und einer generellen Verschiebung von Niederschlagsmengen in das Winterhalbjahr.

Für die Überprüfung der langfristigen Entwicklungen des Grundwasserstands wurden Daten von 312 Messstellen des Hydrografischen Dienstes ausgewertet. Die Auswahl der Messstellen erfolgte nach den Gesichtspunkten, ob einerseits eine längerfristige Aufzeichnung von zumindest 20 Jahren vorliegt und ob für die Messstellen auch Temperaturaufzeichnungen verfügbar sind. Für rund die Hälfte der Messstellen reichen die Aufzeichnungen 40 oder 50 Jahre zurück, für die meisten übrigen Messstellen zumindest rund 20 Jahre. Insgesamt wurden 35 Messstellen wegen lückenhafter oder nicht plausibler Daten ausgeschieden, sodass die Untersuchung anhand von 277 Datensätzen durchgeführt wurde.

Abb. 1 zeigt im Detail die Veränderungen der einzelnen Grundwasserpegel innerhalb von 20 Jahren. Die dargestellte Veränderung beruht auf allen zuvor beschriebenen langjährigen und plausiblen Datenreihen von Grundwassermessstellen (20 Jahre oder länger) und ist aus der Erstellung einer linearen Trendgeraden über die gesamt verfügbare Aufzeichnungsdauer der jeweiligen Messstellen errechnet.

Steigende und fallende Grundwasserpegel halten sich in etwa die Waage, wobei die Anzahl der steigenden Pegel leicht überwiegt. Auch die Größenordnung der Anstiege und Rückgänge ist vergleichbar. Einzige Ausnahme ist ein Pegel im südlichen Wiener Becken, der einen Anstieg von knapp 1,7 $\mathrm{m}$ in 20 Jahren aufweist.

Anders sieht die Entwicklung der Schüttungen bei den verfügbaren Quellmessstellen aus (Abb. 2). Für 50 der 82 Messstellen reichen die Aufzeichnungen zumindest 10 Jahre zurück und weisen plausible Werte auf. Für die Analysen wurden auch nur Messstellen herangezogen, die weitestgehend lückenlose Aufzeichnungen über 10 Jahre haben. Dargestellt ist die prozentuelle Veränderung pro Jahr, die aus der Erstellung einer linearen Trendgeraden über die gesamt verfügbare Aufzeichnungsdauer der jeweiligen Messstelle errechnet wurde.

Es ist ersichtlich, dass knapp über 30 der 50 untersuchten Quellmessstellen eine abnehmende Schüttung aufweisen, und dass die höchsten Abnahmen deutlich größer sind als die höchsten verzeichneten Zunahmen. Beachtlich ist des Weiteren, dass es sich dabei um die Veränderungen pro Jahr handelt und jedem Datensatz mindestens ein Beobachtungszeitraum von 10 Jahren zur Durchschnittsbildung zugrunde liegt.

Zusammenfassend kann für die Veränderungen der Ressourcenquantität Folgendes festgehalten werden:

- Die Grundwasserstände weisen mit den jahresdurchschnittlichen großräumigen Niederschlagsbetrachtungen zumindest teilweise eine (zeitlich verschobene) Korrelation auf.

- Die Quellschüttungen zeigen sehr hohe räumliche und zeitliche Variabilitäten. Korrelationen mit jahresdurchschnittlichen großräumigen Niederschlagsbetrachtungen können für die Quellschüttungen nicht 
gefunden werden. Hier bedarf es räumlich und zeitlich hochaufgelöster Daten.

- Bei den Minima und Maxima der Ressourcenverfügbarkeiten sind durch den Klimawandel zeitliche Verschiebungen zu erwarten.

- Bei den Quellschüttungen ist innerhalb der letzten 10 Jahre mehrheitlich ein deutlicher Rückgang festzustellen.

- Mögliche negative Auswirkungen von Trocken- und Hitzeperioden auf die Verfügbarkeit einzelner Ressourcen werden durch die zunehmende Wahrscheinlichkeit von früheren Maximalständen und früheren Rückgängen der Grundwasserkörper und Quellschüttungen im Jahresverlauf erhöht.

- Höhere Wintertemperaturen, abnehmende Schneeniederschläge und dadurch schnellere und stärkere Oberflächenabflüsse im Winter könnten die Grundwasserneubildung negativ beeinflussen.

\subsubsection{Einflüsse auf die Ressourcenqualität}

Das EU-Projekt CC-WaterS (2012) befasst sich unter anderem auch mit der Auswirkung des Klimawandels auf die Landnutzung und in weiterer Folge mit den Einflüssen einer veränderten Landnutzung auf die Wasserressourcen. Die Ressourcenqualität kann demnach auf verschiedene Arten beeinträchtigt werden. Im Wesentlichen geht es aber immer um eine Mobilisierung von zumeist im Oberboden befindlichen Substanzen (Huminstoffen, Nitrat, Pflanzenschutzmittel, Mikroorganismen oder mineralischen Partikeln) und deren Einschwemmung in das Grundwasser. Die Mechanismen der Kontamination sind

- Herabsetzung der Filterwirkung der oberen Bodenschichten,

- Verletzung der Bodenschichten durch Bodenerosion infolge von Starkniederschlägen, unsachgemäßer Bodenbearbeitung oder Windwurf von Bäumen,

- Trockenrisse infolge langer Trockenperioden und hoher Evapotranspiration und

- Eintrag infolge von Niederschlägen auf verletzte Bodenschichten.

Durch den Klimawandel ist mit erhöhten Temperaturen und dadurch weniger Schneeniederschlag und mehr Regenniederschlag sowie mit einer $\mathrm{Zu}$ - nahme der Niederschlagsmenge aus Starkniederschlägen (Haslinger et al. 2015) zu rechnen. Dadurch erhöht sich die Gefahr der Bodenerosion durch Oberflächenabfluss. Des Weiteren ist mit längeren, häufigeren oder intensiveren Trockenperioden und höherer Evapotranspiration $\mathrm{zu}$ rechnen. Dadurch können vermehrt Trockenrisse im Boden entstehen oder die Widerstandsfähigkeit einzelner Pflanzenarten durch Trockenschäden herabgesetzt werden. Für bewaldete Quellschutzgebiete ist das Absterben einzelner Bäume infolge veränderter Bedingungen oder der Veränderung der Schädlinge relevant. Die Folge kann verstärkte Humusund Bodenerosion und Herabsetzung der Filterwirkung der oberen Bodenschichten sein, bevor eine natürliche Veränderung der Vegetation wieder die Schutzfunktion übernimmt.

Ob durch den Klimawandel auch von häufigeren Unwettern mit Sturm auszugehen ist, kann nicht eindeutig vorhergesagt werden. Höhere Regenmengen durch höhere Temperaturen (z. B. bei $\mathrm{Vb}$-Wetterlagen) führen aber jedenfalls eher zur Verletzung der oberen Bodenschichten durch vermehrten Oberflächenabfluss. Das Risiko der Bodenerosion ist dabei am höchsten, wenn der Boden entweder besonders trocken ist (die Benetzung der trockenen Oberflächen erfolgt dann nicht schnell genug, um Infiltration $\mathrm{zu}$ ermöglichen) oder wenn der Boden schon wassergesättigt ist und keine weitere Infiltration mehr möglich ist. Im Fall einer Zunahme von extremeren Wetterereignissen und stärkeren Stürmen ist dementsprechend auch ein vermehrter Windwurf in bewaldeten Quellschutzgebieten von Bedeutung (CC-WaterS 2012).

Hinsichtlich der chemischen Belastung des Grundwassers weist der NGP (2015) auf den Zusammenhang der Grundwasserqualität mit Stoffeinträgen aus der Landwirtschaft und Verdünnungseffekten durch Niederschläge hin. Speziell in Regionen mit niedrigen Niederschlagsmengen (Ostösterreich) komme es durch die sehr geringe Verdünnung der Stoffeinträge zu erhöhten Konzentrationen im Grundwasser. Die Anzahl der Messstellen in voraussichtlichen Maßnahmengebieten, welche die Qualitätsziele bezüglich Nitrat überschreiten, ist zunehmend. Ein Grundwasserkörper, der bereits im NGP 2009 als voraussichtliches Maßnahmengebiet eingestuft war, zeigt im NGP 2015 zudem einen anhaltenden, signifikant steigenden Trend für Nitrat. Die österreichweite Anzahl aller einzelnen Messstellen, die durch den Parameter Pestizide insgesamt gefährdet sind, hat sich vom NGP 2009 zum NGP 2015 von 26 auf 41 erhöht, obwohl der Umfang der getesteten Messstellen sogar geringfügig reduziert wurde. Ein durch den Klimawandel bedingter, eventuell nötig werdender vermehrter Einsatz z. B. von Pestiziden würde die Qualitätszielverfehlungen jedenfalls verschärfen.

Weitere Auswirkungen auf die Ressourcenqualität sind durch die steigende Temperatur gegeben. Dadurch werden beispielsweise biologische $\mathrm{Ab}$ läufe und chemische Randbedingungen in Grundwasserkörpern beeinflusst. In einer Publikation des Austrian Climate Research Programme ACRP (Wasserwirtschaft 2015) wird dazu ausgeführt, dass die Veränderungen Umsatzprozesse aquatischer Organismen, die Sauerstofflöslichkeit und den pH-Wert betreffen.

Im Forschungsprojekt „StartClim2005.A4“ (Perfler et al. 2006) wird hinsichtlich der Trinkwassernutzung angemerkt, dass die wesentlichen Auswirkungen erhöhter Ressourcentemperaturen die Mikrobiologie des Wassers betreffen. Die Temperatur ist einer der wichtigsten Umweltfaktoren für das Wachstum und das Überleben von Mikroorganismen.

Für Temperaturänderungen gilt generell, dass Mikroorganismen ihre Aktivität mit steigender Temperatur erhöhen - zumindest bis zu einem artenspezifischen Temperatur-Optimum. Für die meisten in der Wasserversorgung relevanten (mesophilen) Mikroorganismen liegt das Temperatur-Optimum zwischen 20 und $40{ }^{\circ} \mathrm{C}$ und somit deutlich höher als die üblichen Grundwassertemperaturen. Unter $10{ }^{\circ} \mathrm{C}$ kommt bei mesophilen Mikroorganismen das Wachstum (Teilungsrate) weitgehend zum Stillstand. Zu den mesophilen Mikroorganismen gehören unter anderem E. coli, Pseudomonas oder Streptococcus. Jede Erhöhung der Ressourcentemperatur würde somit eine Verbesserung der Lebensbedingungen der relevanten Mikroorganismen bedeuten.

Dass die Ressourcentemperaturen im Steigen begriffen sind, lässt sich bereits anhand verfügbarer Messreihen belegen.

Abb. 3 und Abb. 4 zeigen diesbezüglich die Temperaturveränderung von 280 Grundwassermessstellen bzw. 


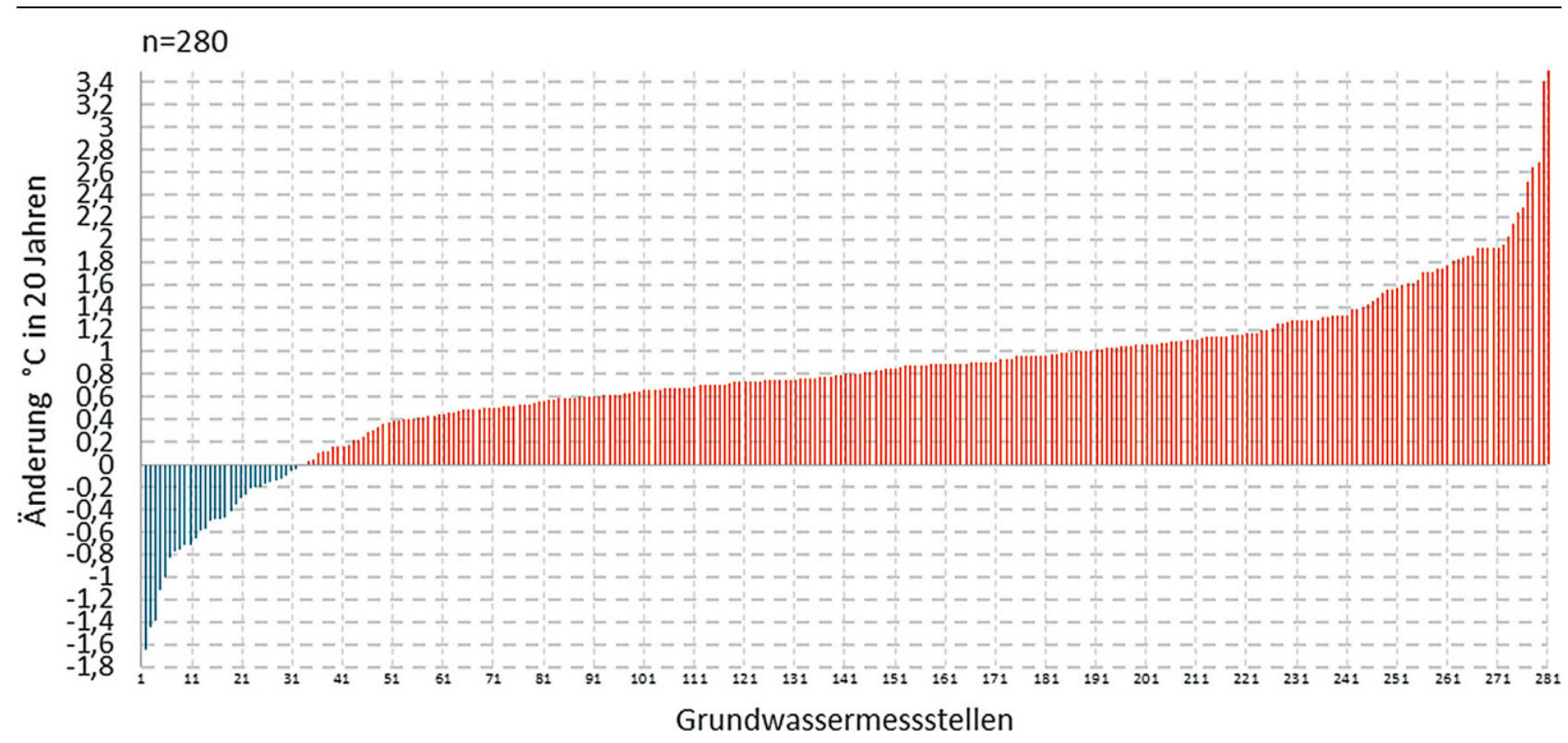

Abb. 3 Änderung der Grundwassertemperatur innerhalb der letzten 20 Jahre (Daten: eHYD 2015, eigene Darstellung)

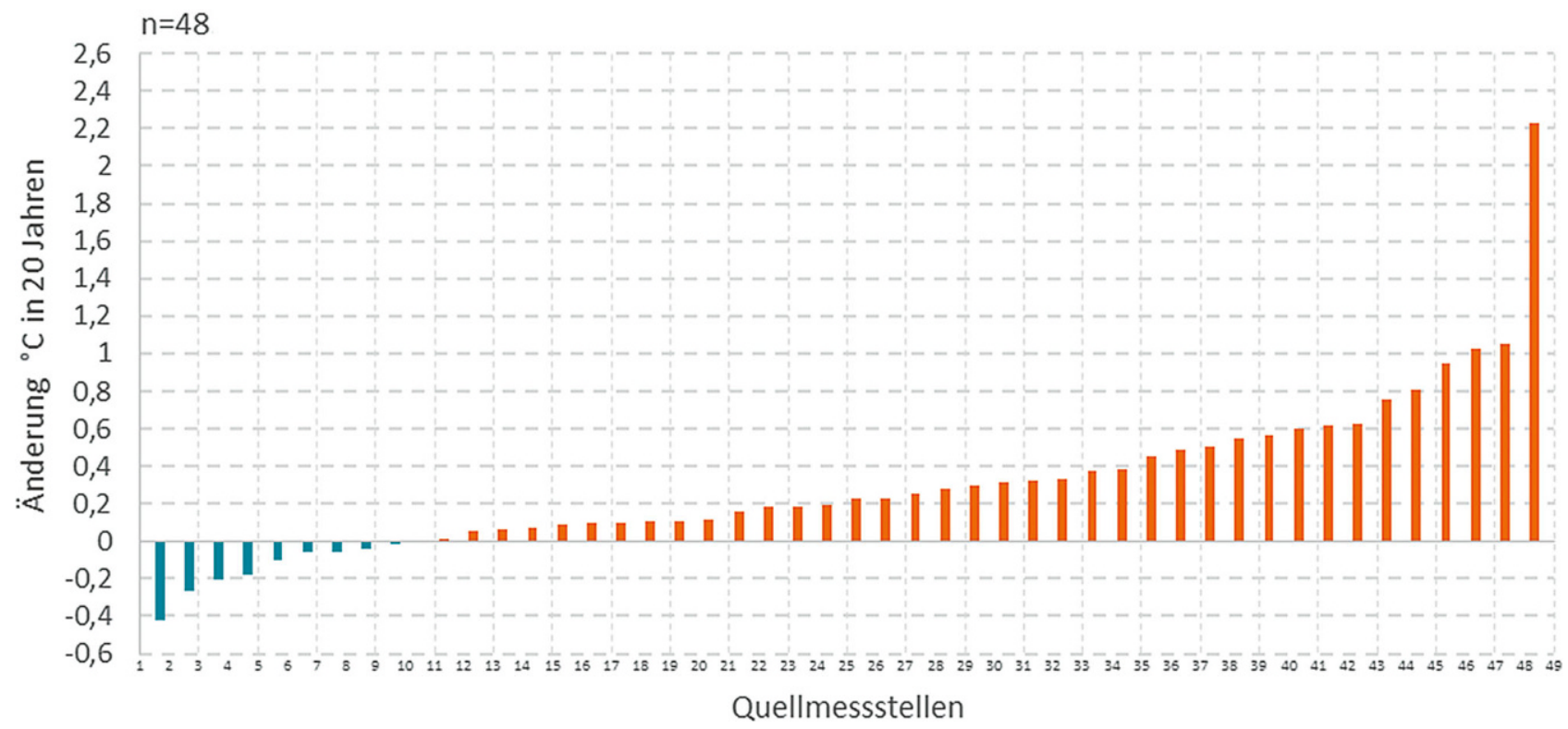

Abb. 4 Änderung der Quellwassertemperatur innerhalb der letzten 20 Jahre (Daten: eHYD 2015, eigene Darstellung)

48 Quellmessstellen innerhalb der letzten 20 Jahre.

Es ist ersichtlich, dass sowohl die Mehrzahl der Grundwasserkörper wie auch der Quellen einen Temperaturanstieg aufweisen.

Die in der Umfrage zur Studie angegebenen maximalen Temperaturen einiger Wasserressourcen nähern sich schon einem für mesophile Mikroorganismen annehmbaren Bereich zum Überleben. Eine tatsächliche mikrobiologische Belastung der Ressource erfolgt natürlich immer nur unter der
Voraussetzung, dass auch die Nährstoffverfügbarkeit gegeben ist und eine primäre Kontamination der Ressource stattgefunden hat.

Zusammenfassend kann festgehalten werden, dass

- die Wassertemperaturen des Großteils aller beobachteten Ressourcen bereits deutlich angestiegen ist und die Erwärmung des Bodens und somit der Grundwässer in den vergangenen Jahren jedenfalls schneller vor sich gegangen ist als die globale Erwärmung, und
- erhöhte Ressourcentemperaturen bedeuten jedenfalls eine Verbesserung der Lebensbedingungen für trinkwasserrelevante (meist mesophile) Mikroorganismen.

\subsection{Bedarfssituation}

\subsubsection{Charakteristik des Wasserverbrauchs}

Hinsichtlich der Bedarfsermittlung ist weniger der durchschnittliche Wasserverbrauch als vielmehr der Spit- 

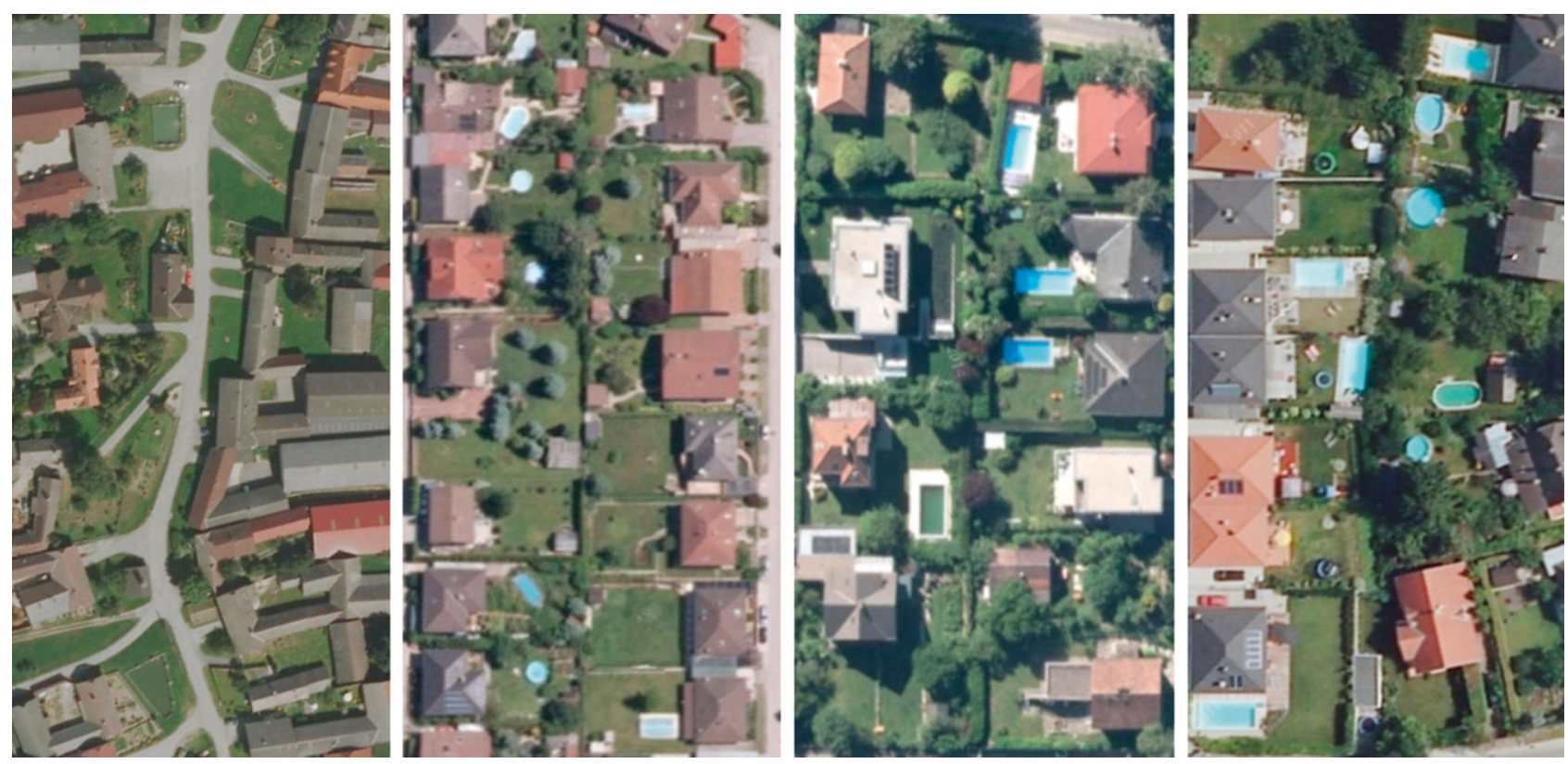

Abb. 5 Verbreitung privater Swimmingpools unterschiedlicher Siedlungsstrukturen (v.l.n.r.): ländlich alter Ortskern, ländliche Siedlungserweiterung, Stadtrand, Speckgürtelgemeinde (Bildquelle: basemap.at)

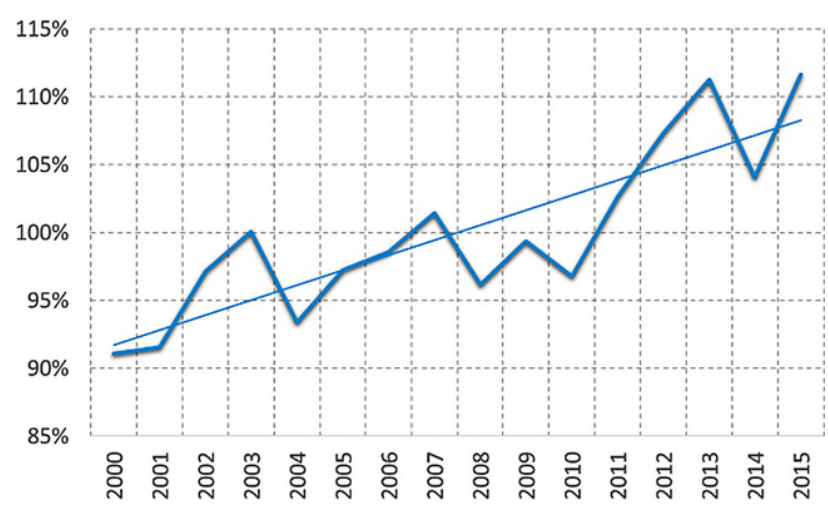

Abb. 6 Verbrauchsentwicklung eines WVU (Datenquelle: Umfragedaten der gegenständlichen Studie)

zenverbrauch für die Bemessung der Versorgungssysteme relevant. Als wesentlicher Auslöser für Spitzenwasserverbräuche wurde bereits in einer früheren Studie (Neunteufel et al. 2013) die Verbrauchergruppe der privaten Haushalte identifiziert. Dies konnte anhand der Umfrageergebnisse zur Studie abermals bestätigt werden.

Die Ursachen für die sommerlichen Spitzenwasserverbräuche sind die verstärkte Bewässerung der Hausgärten und das Nachfüllen von privaten Swimmingpools zur Wassererneuerung und Abkühlung. Die Erstbefüllung privater Swimmingpools ist demgegenüber eher für die Spitzenwasserverbräuche im Mai verantwortlich.
Der Einfluss der sozioökonomischen Situation ist am stärksten anhand der Verbreitung privater Swimmingpools sowie der sonstigen Außenwasserverbräuche in Abhängigkeit von der Grundstücksgröße ersichtlich, während andere Wassernutzungen im Haushalt weniger bis gar nicht mit der der sozioökonomischen Situation zusammenhängen.

Je nach Siedlungsstruktur und Region ist eine unterschiedlich starke Verbreitung privater Swimmingpools erkennbar. Abb. 5 zeigt diesbezügliche Beispiele. Beginnend bei einem ländlichen Ortskern (gänzlich ohne private Swimmingpools) reichen die Varianten bis hin zu einer Speckgürtelgemeinde nahe eines großen Ballungszentrums, wo in einigen Siedlungsteilen rund $60 \%$ der Häuser einen privaten Pool im Garten haben.

Die Kategorie „Poolfüllungen“ ist die am häufigsten explizit genannte Ursache für Spitzenverbräuche in der Umfrage. Betroffen sind dementsprechend insbesondere jene WVU, die in ihrem Versorgungsgebiet einen wesentlichen Anteil an Reihenhäusern oder Ein- und Mehrfamilienhäusern versorgen, die über einen Eigengarten und ggf. Swimmingpools verfügen. Der Anteil der Wassernutzung in Außenbereichen ist dabei bei Einfamilienhäusern, durch die zumeist größeren Gartenflächen, stärker ausgeprägt als bei Reihenhäusern, die im Allgemeinen geringere Gartenflächen aufweisen.

Aufgrund der deutlichen Kopplung des Wasserverbrauchs mit der Temperatur geht auch der Österreichische Sachstandsbericht Klimawandel (APCC 2014) von einer zukünftigen $\mathrm{Zu}$ nahme der Verbrauchskomponenten im Außenbereich (Gartenbewässerung, Befüllung von Pools etc.) aus.

Im Zuge der Umfrage wurden auch Datenreihen der Einspeisemengen einzelner WVU untersucht und beschrieben. Das Beispiel in Abb. 6 zeigt die Entwicklung der Jahresverbrauchsmengen eines WVU mit stark steigendem Gesamtverbrauch aufgrund des Bevölkerungszuwachses und der sozioökonomischen Entwicklung. 


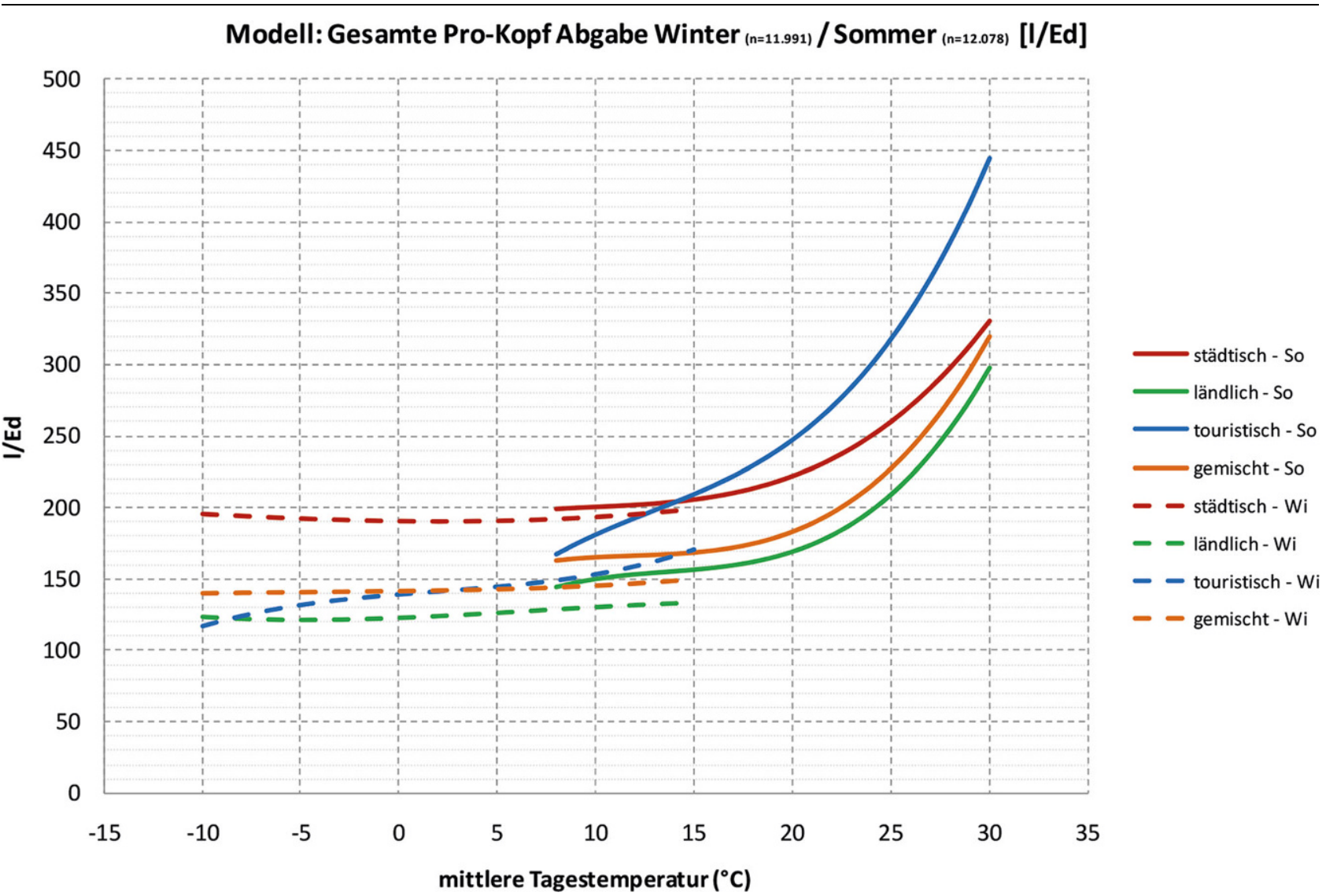

Abb. 7 Modelle für den Einfluss von Temperatur und Jahreszeit auf den Bedarf - differenzierte Ergebnisse nach Strukturgruppen (Grafik: WAVE-Studie, Neunteufel et al. 2013)

Der Einfluss von Tagestemperatur und Trockenheit auf den Wasserverbrauch wurde bereits eindeutig belegt (Neunteufel et al. 2013) und kann in Wasserverbrauchsmodellen dargestellt werden. Abb. 7 zeigt die aus empirischen Daten abgeleiteten Temperaturmodelle für den tagesdurchschnittlichen Wasserbedarf je Einwohner und Tag (l/Ed) differenziert in unterschiedliche Siedlungsstrukturgruppen und unter Berücksichtigung der Jahreszeit (Sommer/Winter).

Für die Aussage, wie stark der Wasserverbrauch mit zunehmenden Temperaturen und häufigeren oder längeren Trockenperioden steigen kann, ist darüber hinaus die Kenntnis über die Ursachen und Zusammensetzung der Spitzenverbräuche nötig.

Abb. 8 zeigt, wie sich die durchschnittliche Zusammensetzung des Wasserverbrauchs von 24 untersuchten Haushalten unter dem Einfluss der Temperatur verändert. Die zusätzlichen Verbrauchsanteile bei hohen Temperaturen entstehen großteils im Außenbereich. Im Innenbereich ist ein Anstieg der Wassermengen für Dusche und WC zu erkennen, während die Nutzung der Badewanne fast gänzlich wegfällt.

Ergänzend ist noch festzuhalten, dass durch den geringer werdenden Sommerniederschlag und vermehrte Hitzeperioden nicht nur private Nutzer ihren Verbrauch steigern werden, sondern auch die Landwirtschaft langfristig ihre Bewässerungskapazitäten ausbauen und den Konkurrenzdruck auf die Ressourcen erhöhen wird.

\subsubsection{Verbrauchssteuerung (Demand Side Management)}

Beispiele einzelner Gemeinden zeigen, dass es gelegentlich Verbote für das Bewässern von Gärten, das Füllen von Schwimmbecken und das Autowaschen geben kann. Bevor es aufgrund von Ressourcenknappheit $\mathrm{zu}$ derartigen Mahnahmen kommt, gilt es Verbrauchsspitzen $\mathrm{zu}$ reduzieren, die durch ein hohes Maß an Gleichzeitigkeit die Versorgungssicherheit gefährden. Die oft gleichzeitig auftretenden, intensiven Wassernutzungen betref- fen dabei wieder insbesondere die $\mathrm{Be}$ füllung von Swimmingpools und die Bewässerung von Gärten und Grünflächen. Dementsprechend muss die Verbrauchssteuerung auch bei diesen Verbrauchsanteilen ansetzen.

Die Art und Wirksamkeit der Verbrauchssteuerung wurde in der Umfrage zur Studie erhoben und thematisch zusammengefasst. Nur knapp ein Drittel der österreichischen Umfrageteilnehmer verwendet Maßnahmen zur Verbrauchssteuerung, und es handelt sich dabei fast ausschließlich um irgendeine Art des Managements (Koordination) der erstmaligen Befüllung der Swimmingpools (privater wie auch öffentlicher Bäder) im Frühjahr. Die Wirksamkeit der Maßnahmen wird unterschiedlich angegeben.

Gesonderte „Poolabgaben“ von Besitzern privater Swimmingpools zu verlangen, wird als kontraproduktiv eingeschätzt, da bei der Einhebung von Sonderabgaben die Bereitschaft der Konsumenten sinkt, sich zusätzlich an Poolfüllungspläne der WVU zu halten. Ein wichtiger Schritt zur Verbrauchs- 


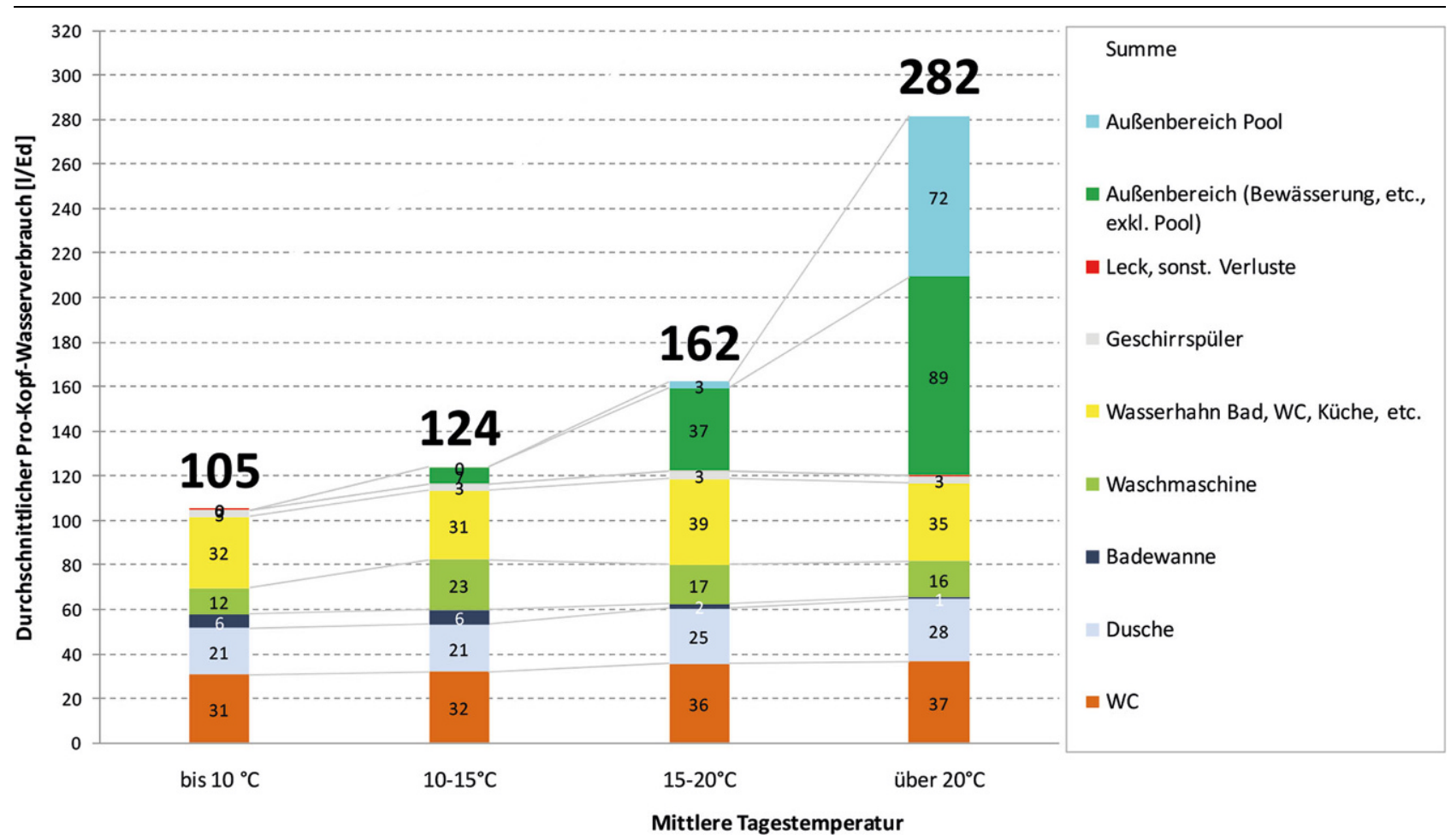

Abb. 8 Einfluss der Temperatur auf die Verbrauchszusammensetzung im Haushalt (Grafik: WAVE-Studie, Neunteufel et al. 2013)

steuerung ist zudem, die Poolfüllungen nur über die Hauswasserzähler zu erlauben und keine Füllungen über Hydrantenanschlüsse $\mathrm{zu}$ ermöglichen auch wenn diese gemessen werden.

Da Nachfüllungen der Pools und Bewässerungen speziell für die Hochsommerspitzen in Rekordsommern verantwortlich sind, liegt es nahe, dass auch hier von vornherein Verbrauchssteuerungen überlegt werden. Derartige Maßnahmen sind jedoch bei keinem Umfrageteilnehmer umgesetzt.

\section{Conclusio und Handlungsoptionen}

Aufgrund der Langlebigkeit und langsamen Anpassung der Wasserinfrastruktur gilt es, ein breites Wissen über die möglichen Szenarien zur Überprüfung der Planungsansätze verfügbar zu haben. Die wichtigsten Kernaussagen lassen sich folgendermaßen zusammenfassen:

- Die Ressourcensituation wird sich durch den Klimawandel langsam aber sicher verändern. Nicht alle Ressourcen werden gleichermaßen bzw. gleich stark betroffen sein. Die Auswirkungen betreffen sowohl quantitative wie auch qualitative Aspekte.

- Nutzungskonflikte werden zunehmen.
- Eine regional integrierte Wasserversorgungsplanung soll daher insbesondere eine Ressourcennutzungsplanung beinhalten und die Bestandsaufnahmen verfügbarer Ressourcen, bestehender Wassernutzungen und Prognosen des zukünftigen Wasserbedarfs umfassen.

- Der Wasserbedarf, insbesondere die Bedarfsspitzen, werden steigen. Von dem intensivierten Wasserbedarf werden insbesondere jene WVU betroffen sein, die in ihrem Versorgungsgebiet einen wesentlichen Anteil an Reihenhäusern oder Ein- und Mehrfamilienhäusern versorgen, die über einen Eigengarten und ggf. Swimmingpools verfügen. Speziell kleinere Versorgungseinheiten werden durch Verbrauchsspitzen stärker unter Druck geraten.

Die Handlungsoptionen für Wasserversorger sind nachfolgend stichwortartig zusammengefasst:

- Ressourcennutzungsplanung zur Prioritätenfestlegung von Anpassungsmaßnahmen und Bewusstseinsschaffung für rechtzeitige Anpassung, Beobachtung der Ressourcenentwicklung zur rechtzeitigen Reaktion auf Veränderungen.

- Verbesserter Ressourcenschutz zur Reduktion des Nutzungsdrucks und
Erhöhung der zukünftigen Ressourcenverfügbarkeit, Steigerung der Ausfallssicherheit und Resilienz, Alternativressourcen zur Erhöhung der Versorgungssicherheit, Aufbereitung um Notressourcen verfügbar zu machen.

- Infrastrukturmanagement und Rehabilitationsmaßnahmen zur Werterhaltung der Infrastruktur und Verbesserung der wirtschaftlichen Nachhaltigkeit.

- Verbrauchssteuerung (demand side management) zur Verminderung der Spitzenverbräuche.

Danksagung Die Erstellung der Studie erfolgte im Auftrag der ÖVGW und mit Unterstützung des BMLFUW. Als Steering Committee wurde die Studie durch eine vom Auftraggeber eingesetzte Task Force, bestehend aus Vertretern der ÖVGW, des Ministeriums und von Wasserversorgern, begleitet. An dieser Stelle sei den Mitgliedern der Task Force ein herzlicher Dank für das Engagement und die Unterstützung bei der Entstehung der Studie ausgesprochen. Ebenso herzlich bedanken wir uns bei den Wasserversorgungsunternehmen, die an der Umfrage teilgenommen und die Studie mit zusätzlichen Daten und wertvollen Informationen unterstützt 
haben. Besonderer Dank gilt auch den zuständigen Beamten der Landesregierungen für die ausführlichen Gespräche, die im Zuge der Experteninterviews geführt wurden und für deren Unterstützung der Studie mit regionalen Erfahrungen und Daten.
Open access funding provided by University of Natural Resources and Life Sciences Vienna (BOKU).

Open Access Dieser Artikel wird unter der Creative Commons Namensnennung 4.0 International Lizenz (http:// creativecommons.org/licenses/by/4. 0/deed.de) veröffentlicht, welche die
Nutzung, Vervielfältigung, Bearbeitung, Verbreitung und Wiedergabe in jeglichem Medium und Format erlaubt, sofern Sie den/die ursprünglichen $\mathrm{Au}$ tor(en) und die Quelle ordnungsgemäß nennen, einen Link zur Creative Commons Lizenz beifügen und angeben, ob Änderungen vorgenommen wurden.

\section{Literatur}

APCC (2014): Österreichischer Sachstandsbericht Klimawandel 2014 (AAR14). Austrian Pane on Climate Change (APCC), Verlag der Österreichischen Akademie der Wissenschaften, Wien, Österreich, 1096 Seiten. ISBN 978-3-7001-7699-2 CC-WaterS (2012): Climate Change and Impacts on Water Supply, http://www.ccwaters.eu/ (Abrufe im Zeitraum Dez. 2015 bis Feb. 2016)

eHYD (2015): Messstellendaten (Grundwasse und Quellwasser); http://ehyd.gvat/ (Abruf im Zeitraum 11-2015 bis 12-2015)

Formayer H., Nadeem, I. Anders, I. (2015): Climate Change Scenario: from Climate Model Ensemble to local indicators, Chapter 5 in Steininger et al 2015 .

Haslinger, K., Schöner, W., Anders, I. (2015): Future drought probabilities in the Greater Alpine Region based on COSMO-CLM experiments - spatial patterns and driving forces; Meteorologische Zeitschrift, PrePub DOI 10.1127/ metz/2015/0604

Neunteufel, R., Richard, L., Perfler, R. (2013) Wasserverbrauch und Wasserbedarf; Auswe tung empirischer Daten zum Wasserverbrauch, Herausgeber: Bundesministerium für Land- und
Forstwirtschaft, Umwelt und Wasserwirtschaft, Sektion VII Wasser, Wien 2013

Neunteufel, R., Schmidt, B., Perfler, R. (2016:) Wasserversorgung im Jahre 2015 - Erfahrungen und Ausblick Studie im Auftrag der ÖVGW, Wien 2016; http://www.ovgw.at/media/medialibrary/ 2016/04/Studie_Wasserversorgung_2015.pdf (Abruf am 31.1.2017)

NGP (2015): Entwurf des NGP (Nationaler Gewässerbewirtschaftungsplan) 2015, BUNDESMINISTERIUM FÜR LAND- UND FORSTWIRTSCHAFT, UMWELT UND WASSERWIRTSCHAFT Sektion IV Wasserwirtschaft, Wien 2015

Perfler, R., Unterwainig, M., Formayer, $\mathbf{H}$ (2006): StartClim - Auswirkungen von Extremereignissen auf die Sicherheit der Trinkwasserversorgung in Österreich; StartClim2005.A4 Teilprojekt von StartClim2005 „Klimawande und Gesundheit“, URL: http://www.austroclim. at/startclim/

Solomon, S., Qin, D., Manning, M., Alley, R. B. Berntsen, T., Bindoff, N. L., Chen, Z., Chidthaisong, A., Gregory, J. M., Hegerl, G. C., Heimann, M. Hewitson, B., Hoskins, B. J., Joos, F., Jouzel, J., Kattsov, V., Lohmann, U., Matsuno, T. Molina, M., Nicholls, N., Overpeck, J., Raga,
G, Ramaswamy, V, Ren, J., Rusticucci, M., Somerville, R., Stocker, T. F., Whetton, P., Wood R. A. and Wratt, D. (2007): Technical Summary. In: Climate Change 2007: The Physical Science Basis. Contribution of Working Group I to the Fourth Assessment Report of the Intergovernmental Panel on Climate Change [Solomon, S., D. Qin, M. Manning, Z. Chen, M. Marquis, K.B. Averyt, M. Tignor and H.L. Miller (eds.)]. Cambridge University Press, Cambridge, United Kingdom and New York, NY, USA

Steininger, K., König, M., Bednar-Friedl, B., Kranzl, L., Loibl, W., Prettenthaler, F. (Editors) (2015): Economic Evaluation of Climate Change - Development of a Cross-Sectoral Framework and Results for Austria (COIN), ISSN 23520698 ISSN 2352-0701 (electronic) ISBN 978-3 319-12456-8, ISBN 978-3-319-12457-5 (eBook), DOI 10.1007/978-3-319-12457-5, Springer Cham Heidelberg New York Dordrecht London

Wasserwirtschaft (2015): BERICHTE ZUR KLIMAFOLGENFORSCHUNG: Wasserwirtschaft, Herausgeber: Klima- und Energiefonds, www. klimafonds.gv.at 